\title{
Adaptação de Metadados para Repositórios de Objetos de Aprendizagem
}

\author{
Liane Margarida Rockenbach Tarouco ${ }^{1}$ \\ Marcelo Augusto Rauh Schmitt ${ }^{2}$
}

\begin{abstract}
Learning objects proliferation allied to lack of an organization in its storage causes difficulties to locate and to reuse this type of material, opposing one of the main goals of the learning object proposal. Referatories and repositories have been created and organized in federations of repositories and this process is described in this paper with examples from a case study related to an implemented repository. This article describes the studies and experiments aiming at the implantation of an Learning Object Repository based on LOM and subsequent steps to integration with Moodle environment to allow the search and access to the content of the repositories from inside this virtual environment of learning.
\end{abstract}

Keywords: learning object repository, referatories, metadata

Resumo. A proliferação de objetos de aprendizagem aliada à falta de uma organização no seu armazenamento ocasiona dificuldades para localizar e reusar este tipo de material, contrariando uma das principais metas da proposta de objetos de aprendizagem. Referatórios e repositórios tem sido criado e organizados em federações de repositórios e este processo é descrito neste artigo sendo exemplificado a partir de um estudo de caso de um repositório implementado. Este artigo descreve os estudos e experimentos visando a implantação de um Repositório de Objetos de Aprendizagem que usa como base os metadados do LOM e os passos subseqüentes para viabilizar sua integração com o Moodle com vistas a facilitar a busca e acesso ao conteúdo do repositório a partir deste ambiente virtual de aprendizagem.

Palavras-chave: repositórios de objetos de aprendizagem, referatórios, metadados

\section{Introdução}

A tecnologia de informática e comunicação atualmente permite criar material didático usando multimídia com interatividade que tornam mais efetivos os ambientes de ensinoaprendizagem apoiados nas TICs. No entanto, o projeto e desenvolvimento desses recursos, mesmo considerando o uso de linguagens de autoração, demandam muito esforço e envolvem grandes investimentos em recursos humanos e financeiros.

Isto ensejou o desenvolvimento da estratégia de orientar sua construção na metodologia orientada a objetos. Os recursos educacionais construídos segundo esta estratégia foram denominados objetos educacionais (learning objects) e organismos de padronização como o IEEE (1484.12.1-2002 Standard for Learning Object Metadata) e ISO (SC 36 WG 2 - Information Technology for Learning, Education, and Training)

\footnotetext{
${ }^{1}$ Professora CINTED-UFRGS e PGIE-UFRGS. Email: liane@penta.ufrgs.br

${ }^{2}$ Professor do Instituto Federal do Rio Grande do Sul Email: schmitt@etcom.ufrgs.br
} 
têm grupos trabalhando na elaboração de propostas para sua estruturação e categorização (metadados). Objetos educacionais são mais eficientemente aproveitados quando organizados em uma classificação de metadados e armazenados em um repositório integrável a um sistema de gerenciamento de aprendizagem (Learning Management System). A adoção de padrões abertos para este fim é desejável uma vez que o rápido avanço da tecnologia leva à possível substituição de plataformas de gerenciamento de aprendizagem com maior rapidez do que a desatualização e/ou obsolescência de um objeto educacional os quais podem ser atualizados e continuar a ser reusados em outro contexto. A estratégia de adotar padrões abertos também tem como objetivo alcançar independência de plataforma onde os objetos vão ser exibidos/executados permitindo o uso de diferentes sistemas operacionais e plataformas de hardware.

\section{Padrões de Metadados}

O metadado de um objeto educacional descreve características relevantes que são utilizadas para sua catalogação em repositórios de objetos educacionais reusáveis que posteriormente podem ser recuperados através de sistemas de busca ou utilizados através de Learning Management Systems (LMS) para compor unidades de aprendizagem as quais, por sua vez vão ser utilizadas em cursos.

Organismos de padronização como o IEEE (1484.12.1 Standard for Learning Object Metadata) e ISO (SC 36 WG 2 - Information Technology for Learning, Education, and Training) têm grupos trabalhando na elaboração de propostas para sua estruturação e categorização (metadados). A especificação de metadados da norma IEEE 1484.12.1 (Learning Objects Metadata) define atributos agrupados em nove categorias para descrição de um objeto educacional. A utilização dos atributos é opcional, ou seja, uma estrutura de metadados, em conformidade com a norma, pode não conter todos os atributos (IEEE/LTSC, 2002). Outros padrões, derivados do padrão LOM, normalmente por simplificação, foram propostos por entidades e associações de instituições. A ADL definiu o padrão SCORM que inclui uma descrição de objetos de aprendizagem usando um conjunto de metadados derivado do LOM (ADL 2009). Projetos de escopo nacional como o CANCORE do Canadá ou ARIADNE na Europa definiram também outros subconjuntos do LOM. Até mesmo para cursos no ambiente Moodle foi definido um MoodleCore (Moodle 2008).

Por outro lado o padrão Dublin Core é um esquema de metadados que visa descrever objetos digitais, tais como, videos, sons, imagens, textos e sites na web (Donohue 2010). O Dublin Core é um padrão de metadados, composto por 15 elementos, planejado para facilitar a descrição de recursos eletrônicos não tendo sido criado inicialmente para representar objetos de aprendizagem. Seu uso em bibliotecas digitais tem crescido muito a nível nacional e internacional sendo a opção prevista no software DSpace, que é uma das opções mais usadas para suporte a este tipo de serviço.

\section{Referatórios e Repositórios de Objetos de Aprendizagem}

Embora um ambiente virtual de aprendizagem possa conter os objetos de aprendizagem, organizados em um simples sistema de arquivamento interno ao curso ou comum a todos os cursos em um dado ambiente, também é possível implantar um sistema de suporte à gestão de conteúdo educacional através de um serviço específico de Repositórios de Objetos de Aprendizagem (LOR - Learning Object Repository) que 
são espaços que permitem o armazenamento e a gestão do uso e a reutilização destes objetos. Harman (2007) define um repositório de objetos de aprendizagem como um catálogo digital que facilita a pesquisa por objetos de aprendizagem. Este tipo de sistema deve permitir:

- o armazenamento propriamente dito;

- o controle de versões e de publicação;

- a busca dos objetos a partir de suas características;

- o controle de acesso;

- a avaliação dos objetos.

O sistema de catalogação pode usar uma taxonomia padronizada (LOM, IMS, SCORM etc...) ou mesmo algum outro modelo de dados. Através do sistema de consulta e acesso o usuário (professor/aluno buscando conteúdo digital apoiar atividades de ensino/ aprendizagem) pode localizar um conteúdo apropriado para o uso pretendido. Inspecionando os metadados o usuário pode avaliar se o material tem características que atendam sua necessidade e, em caso positivo, pode recuperar o conteúdo para uso, reuso ou reciclagem. O repositório pode conter apenas o endereço (URL) da localização efetiva do conteúdo ou pode conter o próprio material educacional catalogado. No primeiro caso este tipo de sistema pode ser também designado como referatório pois contem apenas as referências aos objetos de aprendizagem

Existem vários exemplos atuais de repositórios bastante conhecidos, a nível internacional, tais como:

- MERLOT - http://www.merlot.org

- Le@rning Federation - http://www.thelearningfederation.edu.au

- eduSource - http://www.edusource.ca/

- National Learning Network - http://www.nln.ac.uk/

- Jorum - http://www.jorum.ac.uk/

Em alguns deles, além do acesso via web é também oferecido acesso via um API (Application Program Interface) tal como no caso do Jorum e mesmo do MERLOT. Este tipo de interface permite que a pesquisa e o acesso a objetos de aprendizagem seja efetuado diretamente a partir de um ambiente virtual de aprendizagem. Com este tipo de serviço, um professor que estivesse preparando uma unidade de aprendizagem poderia realizar uma busca e selecionar objetos de aprendizagem que poderiam então ser impostados para o ambiente ou apenas referenciados permanecendo o objeto de aprendizagem no repositório, o que evitaria a duplicação de material quando usado em diversas turmas, por exemplo.

\subsection{CESTA na fase referatório}

O projeto CESTA - Coletânea de Entidades de Suporte ao uso de Tecnologia na Aprendizagem foi idealizado e implementado em 2003, com vistas a sistematizar e organizar o registro dos objetos educacionais que vinham sendo desenvolvidos pela equipe do Pós-Graduação Informática na Educação e do CINTED - Centro Interdisciplinar de Novas Tecnologias na Educação da UFRGS (TAROUCO 2003). Uma especificação inicial de metadados para os objetos educacionais foi elaborada e a partir da mesma um serviço de registro e catalogação de objetos educacionais foi colocado em operação, usando como base um servidor LDAP (Lighweight Directory Access Protocol). O processo de seleção dos metadados foi baseado na análise das especificações de metatados para os objetos educacionais elaboradas pelo Learning 
Technologies Group do IEEE, e com base nesta seleção foi construída uma especificação com atributos para descrever os objetos educacionais. A especificação de metadados criada para a categorização dos objetos educacionais no repositório foi elaborada em consonância com a norma IEEE 1484.12.1 (Learning Objects Metadata) proposto pelo IEEE Learning Technology Standards Committee (LTSC). A norma especifica atributos agrupados em 9 categorias para descrição de um objeto educacional cujo uso é opcional.

Para a implementação do sistema de cadastramento dos objetos educacionais no CESTA, foram inicialmente utilizadas cinco categorias na especificação dos metadados: geral, ciclo de vida, técnica, educacional e direitos. A preocupação com reduzir a quantidade de metadados utilizados na catalogação dos objetos de aprendizagem deriva da constatação de que em se adotando uma filosofia de autoarquivamento, onde os próprios autores registram e depositam os objetos de aprendizagem que produzem, é necessário facilitar o trabalho de registro. Diversos estudos que investigaram os metadados utilizados em repositórios mostram que é pequeno o conjunto dos que são efetivamente usados e completados pelos usuários do repositórios (Frieses 2004).

O CESTA em sua versão atual contém apenas os endereços de objetos de aprendizagem, tratando-se, pois, de um referatório. A proposta do projeto é apoiada na idéia de auto-arquivamento, ou seja, os autores podem solicitar cadastro no sistema e a partir deste momento estão autorizados a incluir registros e a atualizar os registros por eles incluídos. Todavia, constatou-se que, ao longo do tempo alguns endereços de objetos tornam-se obsoletos em decorrência de transferência para servidores diferentes ou de mera desativação sem que os responsáveis pelos objetos realizem e atualização no registro relacionado com este objeto no referatório. A experiência mostrou que há vantagens em preservar os recursos educacionais, armazenando alguma cópia em um repositório. Para permitir armazenar os objetos e seus metadados, foi iniciado um processo de atualização do CESTA visando ampliar seu escopo inicial. A nova versão do sistema, intitulada CESTA2 foi delineada com vistas oferecer a capacidade de armazenar os metadados, os objetos e, adicionalmente, buscou-se viabilizar outras funcionalidades tais como permitir a coleta de metadados mediante o uso do protocolo OAI-PMH (OAI 2002)

\subsection{Moodle como repositório}

Um ambiente virtual de aprendizagem também pode prover funções de apoio ao armazenamento de objetos de aprendizagem. O ambiente Moodle foi também analisado com vistas a investigar os mecanismos que oferece para funcionar como um LOR. Na instalação padrão, o Moodle não apresenta funcionalidades apropriados provendo apenas um sistema de armazenamento de arquivos organizados em diretórios, internos a cada curso ou comuns a todos os cursos de um dado ambiente. Assim, cada curso possui os seus próprios objetos que são acessíveis apenas pelos usuários cadastrados no curso. Para que um material seja utilizado em curso diferente há necessidade de importá-lo de um curso para o outro. Isto apresenta dois problemas: não há uma ferramenta de busca para que professores ou desenvolvedores encontrem materiais que lhes interesse e ocorre uma duplicação de dados, pois os objetos importados são copiados de um curso para outro. O MrCute (Moodle Repository Create Upload Tag Embed) é um novo módulo desenvolvido para o Moodle pela equipe da Worcester College of Technology que permite incorporar ao Moodle funcionalidades de um repositório (MrCute 2008) 
O módulo MrCute (versão 2) permite carregar conteúdos educacionais empacotados no formato do padrão IMS (Global 2009). Como resultado, um pacote IMS carregado para o repositório MrCute pode ficar acessível no ambiente Moodle, para todos os cursos, independentemente do curso em que foi carregado. $O$ armazenamento do pacote (arquivo zipado) é feito em uma parte da estrutura de arquivos do Moodle, externa a qualquer curso individual e pode ser configurado para ser compartilhado ou não. Isto permite que um professor, cadastrado naquele servidor Moodle, utilize a busca (usando palavras chave ou categorias) para localizar conteúdos apropriados e incorporá-los a um curso. Além disso, o módulo permite a criação de pacotes IMS a partir de conteúdos já criados (por composição). A solução, todavia, é um tanto restritiva porque os mecanismos de busca e utilização restringem-se ao ambiente Moodle no qual o módulo foi instalado. Para permitir repositórios com acesso mais universais integrados ao Moodle, estão sendo desenvolvidos, pela comunidade de desenvolvedores do Moodle, plugins para repositórios externos existentes. A versão Moodle 2.0, que deve ser disponibilizada em julho de 2010, tem prevista uma nova funcionalidade para que, deste ambiente seja possível interagir com repositórios de objetos de aprendizagem usando a própria interface do Moodle. (Moodle 2010).

Assim, usando apenas o módulo MrCute2, atualmente disponível, é possível trazer para o repositório conteúdos externamente desenvolvidos. Também é possível construir um conteúdo educacional usando o próprio Moodle, mediante o uso de ferramentas como a denominada Livro, que é um módulo opcional, que facilita a autoria de conteúdo estruturado em capítulos além de possibilitar a exportação do conteúdo produzido com um empacotamento compatível com o padrão IMS. Isto permite sua transferência para o próprio repositório disponibilizado internamente no Moodle, pelo MrCute, ou para um repositório de objetos de aprendizagem independente, como o Jorum que é mantido pelo Joint Information Systems Comitteé na Inglaterra (JISC 2010). Estas duas funcionalidades, Livro exportado em padrão IMS e MrCute para gestão de conteúdo, foram testadas pelos autores e em virtude das limitações em relação ao padrão de empacotamento (limitado ao formato IMS), levaram à decisão de investigar outra alternativa para implementar um repositório externo ao Moodle para armazenar os objetos de aprendizagem que pudessem ser empacotados em consonância com outros padrões, tal como o SCORM.

Tendo em vista a limitação imposta pelo módulo MrCute2 passou-se então a investigar outras soluções em termos de sistemas que pudessem ser usados para o repositório tal como BELTZ, JOOMLA, DRUPAL e DSPACE tendo sido o DSPACE a solução escolhida. Este software, em sua versão básica, utiliza o padrão de metadados Dublin Core o que demandou um estudo suplementar visando sua expansão para permitir o uso dos metadados padrão LOM, tal como descrito na próxima sessão.

\section{DSpace como repositório de objetos de aprendizagem}

O DSpace é um sistema de repositório digital desenvolvido para a biblioteca do MIT (Massachusetts Institute of Technology) que tem como objetivos recolher, preservar, gerir e disseminar o produto intelectual dos seus investigadores (Donohue 2009). Ele é o resultado de um esforço conjunto de investigação e desenvolvimento do MIT e da Hewlett-Packard (HP). O DSpace é disponibilizado livremente sob a forma de um produto de código aberto, sendo atualmente uma solução muito disseminada e usada por bibliotecas e instituições acadêmicas em geral para apoiar a implementação de repositórios digitais. Este software é usado na UFRGS no portal denominado LUME 
com o objetivo de preservar e divulgar os documentos digitais da instituição (LUME, 2010).

O DSpace aceita todas as formas de materiais digitais, incluindo arquivos de texto, imagem, vídeo e áudio, o que possibilita custodiar os mais variados tipos de conteúdos, tais como, livros, artigos, relatórios técnicos, working papers, artigos de conferências, e-teses, conjuntos de dados (estatísticos, geoespaciais, etc.), programas de computador, modelos e simulações visuais, etc. O DSpace usa de forma nativa o padrão de metadados DCMI - Dublin Core Metadata Initiative (DCMI, 2008) que foi originalmente idealizado para descrever objetos digitais, tais como, vídeos, sons, imagens, textos e sites na web. O padrão Dublin Core inclui dois níveis: Simples e Qualificado. O Dublin Core Simples apresenta apenas quinze elementos, o Qualificado incluiu, inicialmente, três elementos adicionais (Audiência, Proveniência e Detentor de Direitos), assim como um grupo de refinamentos de elementos (também chamados qualificadores), que detalham a semântica dos elementos de maneira que sejam úteis na descobertas de recursos. O conjunto de elementos de metadados Dublin Core Simples (Core Metadata Element Set, DCMES) contempla os seguintes metadados:
1. Title: Título
6. Contributor: Contribuidor
11. Source: Origem
2. Creator: Criador
7. Date: Data
12. Language: Idioma
3. Subject: Assunto
8. Type: Tipo
13. Relation: Relação
4. Description: Descrição
9. Format: Formato
5. Publisher: Publicador
10. Identifier: Identificador
14. Coverage: Abrangência
15. Rights: Direitos

Após a especificação dos quinze elementos originais, foi iniciado um processo de refinamento dos termos existentes além da definição de novos elementos. Alguns termos adicionais foram identificados e o padrão passou a permitir a incorporação de novos metadados definidos sob demanda pelos usuários. Esta funcionalidade foi aproveitada na busca de uma solução que incorporasse os metadados LOM ao ambiente DSpace, no âmbito do CESTA2.

\subsection{A seleção dos metadados para o DSpace no projeto CESTA2}

A fim de adaptar o projeto CESTA ao software DSpace, foi necessário levar em consideração o desejo de se utilizar vários elementos presentes no padrão LOM (IEEE, 2008) que são importante para a identificação, armazenamento e busca de objetos de aprendizagem e o uso da recomendação Qualified Dublin Core (DCMI, 2008) pelo programa. Fez-se necessário, portanto, realizar um mapeamento entre os dois padrões e, em alguns casos, estender as propriedades definidas para o Qualified Dublin Core, criando elementos específicos para a aplicação.

No que diz respeito aos metadados, decidiu-se utilizar elementos presentes em seis categorias do padrão LOM tal como indicados a seguir.

A) Categoria geral - agrupa informações gerais que descrevem o objeto.

a) Identificador - identificação única do objeto;

b) Título - nome dado ao objeto;

c) Idioma - idioma utilizado no objeto (português, inglês, espanhol, francês);

d) Descrição - descrição textual do conteúdo do objeto;

e) Palavras-chave - palavra(s)-chave descrevendo os tópicos do objeto;

f) Nível de agregação - granularidade do objeto. 
B) Categoria ciclo de vida - descreve as características relacionadas com o histórico e o estado atual dos objetos e todos aqueles que o têm afetado durante sua evolução.

a) Versão - a edição do objeto;

b) Status - estado atual do objeto (rascunho, revisado, editado, indisponível);

c) Papel na contribuição - tipo de contribuição (autor, editor, desconhecido, iniciador, designer gráfico, técnico, provedor de conteúdo, roteirista, designer instrucional, especialista em conteúdo);

d) Entidade que contribuiu - identificação de quem contribuiu para a construção do objeto.

C) Categoria técnica - descreve os requisitos e características técnicas do objeto.

a) Formato - formato de todos os componentes do objeto (MIME types), este atributo pode ser usado para identificar o programa necessário para acessar o objeto;

b) Tamanho - tamanho do objeto em bytes;

c) Localização - URL (Universal Resource Locator) do objeto;

d) Requisitos Tecnológicos - requisitos tecnológicos para uso;

e) Instruções de instalação - descrição de como instalar o objeto;

f) Duração - tempo de duração (utilizado para sons, vídeos, animações).

D) Categoria educacional - descreve as características educacionais e pedagógicas do objeto.

a) Tipo de interatividade - modo predominante de aprendizagem (ativa, expositiva, mista);

b) Tipo de recurso de aprendizagem - tipo específico do objeto (exercício, simulação, questionário, diagrama, figura, gráfico, índice, slide, tabela, teste, experiência, texto, problema, auto-avaliação, palestra);

c) Nível de interatividade - grau de interatividade (muito baixo, baixo, médio, alto, muito alto);

d) Usuário final esperado - tipo de usuário para o qual foi desenvolvido o objeto (professor, autor, aluno, gerenciador);

e) Contexto - ambiente no qual o objeto de aprendizagem pode ser utilizado (infantil, fundamental, médio e superior);

f) Faixa etária - idade do usuário final pretendido;

g) Descrição - comentários sobre a forma de utilização do objeto.

E) Categoria direitos - descreve os direitos de propriedade intelectual e as condições de uso do objeto.

a) Custo - se a utilização do objeto requer pagamento (sim ou não);

b) Copyright e outras restrições - existência de restrições de direito autoral para o uso do objeto (sim ou não);

c) Descrição - comentários sobre as condições de uso do objeto.

F) Categoria relação - define as relações com outros objetos de aprendizagem.

a) Tipo - natureza da relação (“parte de”, "versão de”, “substituído por”);

b) Identificador - rótulo que identifica o objeto relacionado.

São utilizados, ainda, dois elementos que não apresentam correspondência com o padrão LOM: títulos alternativos e áreas do conhecimento. O título alternativo 
corresponde à propriedade “title.alternative” definida no padrão DCMI, 2008 e está presente no DSpace. Para identificar as áreas do conhecimento foi criada uma nova propriedade para o Qualified Dublin Core identificada por “description.knowledgeField".

A tabela 1 apresenta o mapeamento realizado entre o modelo LOM e o Dublin Core, tendo por base os elementos e qualificadores definidos no documento DCMI Metadata Terms (DCMI 2008). Duas destas propriedades não estão definidas no esquema do DSpace embora sejam apresentadas no DCMI: "instructionalMethod" e "audience.educationLevel. Foram, portanto acrescentadas ao registro de metadados.

\begin{tabular}{|c|c|c|}
\hline LOM (identificação numérica) & & Qualified Dublin Core \\
\hline General.Title (1.2) & & title \\
\hline General.Description (1.4) & & description.abstract \\
\hline General.Keyword (1.5) & & subject \\
\hline Technical.Requirement.OrComposite.Name (4.4.1.2) & & relation.requires \\
\hline Educational.LearningResourceType (5.2) & & instructionalMethod \\
\hline Educational.Context (5.6) & & audience.educationLevel \\
\hline $\begin{array}{l}\text { Relation.Resource.Identifier.Entry } \\
\text { Relation.Kind = 'ispartof' }\end{array}$ & para & relation.isPartOf \\
\hline $\begin{array}{l}\text { Relation.Resource.Identifier.Entry } \\
\text { Relation.Kind = 'isversionof' }\end{array}$ & para & relation.isVersionOf \\
\hline $\begin{array}{l}\text { Relation.Resource.Identifier.Entry } \\
\text { Relation.Kind = 'isreplacedby' }\end{array}$ & para & relation.isReplacedBy \\
\hline
\end{tabular}

Tabela 1 - Correspondência do LOM para Qualified Dublin Core

A tabela 2 corresponde ao mapeamento entre elementos do LOM para elementos do Qualified Dublin Core que não estão definidos em DCMI, mas que existem no modelo de dados do DSpace. Para estas propriedades não foi preciso alterar o registro de metadados no DSpace. O metadados autor não é mapeado para a propriedade “creator” do Qualified Dublin Core porque o DSpace utiliza este elemento em situação distinta e as funções de busca avançada e ordenamento por autor ficariam prejudicadas.

\begin{tabular}{|l|l|}
\hline LOM (identificação numérica) & $\begin{array}{l}\text { Propriedades específicas do DSpace } \\
\text { para o Qualified Dublin Core }\end{array}$ \\
\hline General.Identifier.Entry (1.1.2) & identifier.uri \\
\hline General.Language (1.3) & language.iso \\
\hline LifeCycle.Version (2.1) & description.version \\
\hline $\begin{array}{l}\text { LifeCycle.Contribute.Entity (2.3.2) ‘autor’ } \\
\text { LifeCycle.Contribute.Role (2.3.1) }\end{array}$ & contributor.author \\
\hline $\begin{array}{l}\text { LifeCycle.Contribute.Entity (2.3.2) } \\
\text { LifeCycle.Contribute.Role (2.3.1)= 'outro’ }\end{array}$ & contributor.other \\
\hline Technical.Format (4.1) & format.mimetype \\
\hline Technical.Size (4.2) & format.extent \\
\hline Technical.Location (4.3) & identifier.uri \\
\hline
\end{tabular}

Tabela 2 - Correspondência entre elementos do LOM e elementos do DSpace não definidos em DCMI, 2008 
A tabela 3 apresenta propriedades derivadas do LOM, e selecionadas para uso no CESTA2 mas que que não estavam presentes no esquema original do DSpace e nem nas definições DCMI, 2008.

\begin{tabular}{|l|l|}
\hline LOM (identificação numérica) & $\begin{array}{l}\text { Propriedades específicas do CESTA2 para o } \\
\text { Qualified Dublin Core }\end{array}$ \\
\hline General.AggregationLevel (1.8) & type.aggregationLevel \\
\hline LifeCycle.Status (2.2) & description.status \\
\hline Technical.InstallationRemarks (4.5) & description.installationRemarks \\
\hline Educational.InteractivityType (5.1) & type.interactivityType \\
\hline Educational.InteractivityLevel (5.3) & type.interactivityLevel \\
\hline $\begin{array}{l}\text { Educational.IntendedEndUserRole } \\
\text { (5.5) }\end{array}$ & audience.intendedEndUserRole \\
\hline Educational.TypicalAgeRange (5.7) & audience.age \\
\hline Educational.Description (5.10) & type.useDescription \\
\hline Rights.Cost (6.1) & rights.cost \\
\hline Rights.Copyright (6.2) & rights.copyright \\
\hline Rights.Description (6.3) & rights.uri \\
\hline
\end{tabular}

Tabela 3 - Elementos do LOM não existentes originariamente em DCMI, 2008 e no DSpace

\subsection{Configuração do DSpace para uso de LOM}

A partir da definição dos metadados que se deseja utilizar em um repositório hospedado no DSpace, algumas configurações são necessárias. É preciso alterar o registro de metadados, a interface de inclusão e de apresentação dos dados, a interface de busca e a comunicação via OAI/PMH (Open Archives Initiative/Protocol for Metadata Harvesting). A alteração do registro de metadados consiste em adicionar novos elementos e qualificadores ao esquema de dados do DSpace. Esta tarefa pode ser realizada através da interface WEB do software pelo administrador do sistema

A interface de inclusão do DSpace precisa ser modificada uma vez que novos campos devem apresentados para preenchimento no momento da inclusão de novos objetos no repositório. Para acrescentar novos elementos quando um objeto de aprendizagem é inserido, deve-se editar os arquivos de configuração input-forms.xml conforme descrito na seção "Alter submisstion input forms” de DSpace (2010 ) e itemsubmission.xml. No primeiro, deve ser efetivada a definição dos novos campos que deverão ser preenchidos pelo usuário quando do carregamento de um objeto de aprendizagem; no segundo, podem ser alterados os passos desta ação. Se for utilizada uma tradução para outra língua, o arquivo input-forms.xml deverá ter adicionado ao seu nome a língua correspondente (ex: input-forms_pt.xml).

Para adaptar a interface de apresentação permitindo a exibição de elementos novos quando um objeto é apresentado, modifica-se o arquivo dspace.cfg, na seção Web Interface Settings. O mesmo arquivo deve ser alterado para se realizar buscas por valores das propriedades acrescentadas na seção "Modify Search Fields" de DSpace (2010). Por último, a fim de permitir o uso do protocolo OAI/MPH, é necessário utilizar crosswalk plugins, que permitem a tradução dos elementos Qualified Dublin Core para outro padrão de metadados conforme descrito na seção "Crosswalk Plugins" de DSpace (2010). 


\section{Estágio atual e próximas fases da nova versão do CESTA2 e conclusões}

O repositório de objetos de aprendizagem implementado no âmbito do projeto CESTA2 possui atualmente um protótipo operacional em teste, o qual apresenta todos os novos metadados definidos incluídos em seu esquema e uma adaptação das interfaces de inserção, consulta e busca (CESTA 2003).

No projeto, está prevista a operacionalização da interface OAI-PMH de modo a permitir a utilização deste protocolo de coleta de metadados tanto para o DCMI quanto para o LOM com vistas a integrar o repositório na Federação Educa Brasil ( FEB 2010).

Adicionalmente, está sendo testada a nova versão do ambiente virtual de aprendizagem Moodle (2.0) para identificar as alterações necessárias para que seja possível a interação entre o CESTA2 e o Moodle. Pretende-se construir uma solução que propicie interação similar àquela possibilitada pelo MrCute2 em relação ao JORUN, permitindo a busca no repositório a partir do interface do Moodle bem como a importação do objeto selecionado para este ambiente virtual de aprendizagem.

\section{Referências}

ADVANCED DISTRIBUTED LEARNING (ADL). Documentation Suite (SCORM 2004 4th Ed.) 2009. Disponível em: <http://www.adlnet.org> Acesso em: 15 maio. 2010.

ARIADNE. (2000). Alliance of remote instructional authoring and distribution networks for Europe website [On-line]. Disponível em: <http://ariadne.unil.ch/> Acesso em: 15 jul. 2004.

CESTA. Coletânea de Entidades de Suporte ao uso de Tecnologia na Aprendizagem . 2003 Acessível em http://www.cinted.ufrgs.br/CESTA/. AAAcessado em Maio de 2010.

DCMI. DMCI Metadata Terms. Dublin Core Metadata Initiative. 2008. Acessível em http://dublincore.org/documents/2008/01/14/dcmi-terms/ acessado em maio 2010.

Donohue, Tim. DSpace. 2010 Disponível em http://wiki.dspace.org/. Acessado em maio de 2010

DUTRA, Renato Luís de Souza; TAROUCO, Liane Margarida R. Recursos Educacionais Abertos. RENOTE. Revista Novas Tecnologias na Educação, v. 5, p. 1-8, 2007.

FEB. Federação Educa Brasil. 2010. Disponível em http://feb.ufrgs.br/. Acessado em Maio de 2010.

Friesen, Norm. LOM Survey: Final Report. 2004. Disponível em http://learningspaces.org/n/cv.html. Acessado em Maio de 2010.

HARMAN, Keith; KOOHANG, Alex. ed. (2007) Learning objects: standards, metadata, repositories, and LCMS. Santa Rosa, Califórnia: Informing Science Press, 2007.

IEEE. 1484.12.1 LOM data model standard. Learning Technologies Standards Committee. 2002.

IMS. (2000). Instructional management systems project website [On-line]. Disponível em: $<$ http://imsproject.org/imMembers.html $>$

KOOHANG, Alex; HARMAN, Keith. ed. (2007a). Learning Objects: Theory, Praxis, Issues and Trends. Santa Rosa, Califórnia: Informing Science Press, 2007.

LUME. LUME - Repositório Digital da Universidade Federal do Rio Grande do Sul. Disponível em http://www.lume.ufrgs.br/ Acessado em maio de 2010 
LTSC. (2000a). Learning technology standards committee website [On-line]. Disponível em: < http://tsc.ieee.org/> Acesso em: 15 jul. 2004.

Moodle. MoodleCore. 2008. Acessível em http://docs.moodle.org/en/Development:Metadata. Acessado em Maio 2010.

OAI. Open Archives Initiative Protocol for Metadata Harvesting. 2002 Acessível em http://www.openarchives.org/OAI/openarchivesprotocol.html. Acessado em Março de 2010

TAROUCO, Liane. CESTA - Coletânea de Entidades de Suporte ao uso de Tecnologia na Aprendizagem. 2003 . Disponível em http://www.cinted.ufrgs.br/CESTA/. Acessado em março de 2010.

TAROUCO, Liane M. R.; DUTRA, Renato Luis de Souza. Padrões e interoperabilidade. In: Carmem Lucia Prata, Anna Christina Aun de Azevedo Nascimento. (Org.). Objetos de Aprendizagem - Uma proposta de recurso pedagógico. Brasília - DF: MEC/SEED, 2007.

TAROUCO, Liane Margarida R.; SCHMITT, Marcelo Augusto Rauh; RODRIGUES, Alessandra Pereira; VICCARI, Rosa Maria. Gestão colaborativa de conteúdo educacional. RENOTE: Revista de Novas Tecnologias na Educação, Porto Alegre, v. 7, n. 1, p.1-13, 01 jul. 2009. Semestral. Disponível em: http://www.cinted.ufrgs.br/renote/. Acesso em: 01 jul. 2009.

WILLEY, David A. (2002) Connecting learning objects to instructional design theory: A definition, a metaphor, and a taxonomy. Disponível em: <http://reusability.org/read/ chpters/wiley.doc>. Acesso em: 07 out. 2009. 